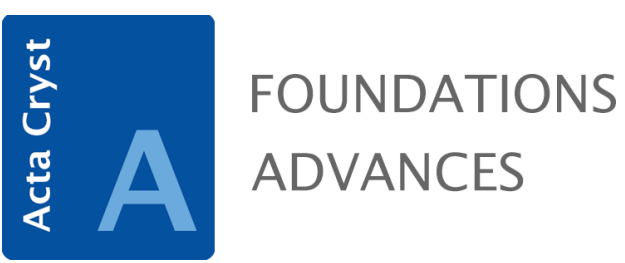

Volume 76 (2020)

Supporting information for article:

Isotopy classes for 3-periodic net embeddings Stephen Power, Igor Baburin and Davide Proserpio 


\title{
ISOTOPY CLASSES FOR 3-PERIODIC NET EMBEDDINGS
}

\author{
I. A. BABURIN, S. C. POWER AND D. M. PROSERPIO
}

\section{Supporting Information}

\section{The proof of Theorem 9.5.}

Proof. Let $\mathcal{M}$ be model net with adjacency depth 1 and a single vertex quotient graph. We show that $\mathcal{M}$ is equivalent to one of the 19 model nets by an elementary affine transformation.

The case $m=3$. In all cases it is clear that $\mathcal{M}$ is equivalent to $\mathcal{M}_{\mathrm{pcu}}$.

The case $m=4$. We consider 4 subcases:

(i) Assume that 3 of the edges of $F_{e}$ are axial edges. Then $\mathcal{M}$ is obtained from $\mathcal{M}_{\mathrm{pcu}}$ by the addition of an additional edge to the motif. If this is a facial edge then, by rotation and translation $\mathcal{M}$ is equivalent to $\mathcal{M}_{\mathrm{pcu}}^{f}$, the model net for the word $a_{x} a_{y} a_{z} f_{x}$. If the extra edge is a diagonal edge then $\mathcal{M}$ is equivalent to $\mathcal{M}_{\mathrm{pcu}}^{d}$.

(ii) Assume that exactly 2 of the 4 edges of $F_{e}$ are axial edges. We may these are $a_{x}, a_{y}$ and we may also assume that neither of the remaining 2 edges is in the $x y$-plane since in this case there would be a triple of coplanar edges in $F_{e}$ and $\mathcal{M}$ would be equivalent to $\mathcal{M}_{\mathrm{pcu}}^{f}$. Suppose first that there is no diagonal edge and so $\mathcal{M}$ is of type $a_{x} a_{y} w$ with $w$ one of $f_{x} f_{y}, f_{x} g_{y}, g_{x} f_{y}, g_{x} g_{y}$. These nets are pairwise equivalent by rotation about the $z$-axis and translation. By an elementary affine transformation they are thus all equivalent to $\mathcal{M}_{\mathrm{pcu}}^{d}$.

Assume on the other hand that only 1 of the 2 extra edges is a facial edge. Translating and rotating we may assume that this edge is $f_{x}$. Also we may assume a noncoplanarity position of the diagonal edge with respect to $f_{x}$ and $a_{x}$, as in Figure 1, since otherwise there is an oriented affine equivalence with the model net for hex.
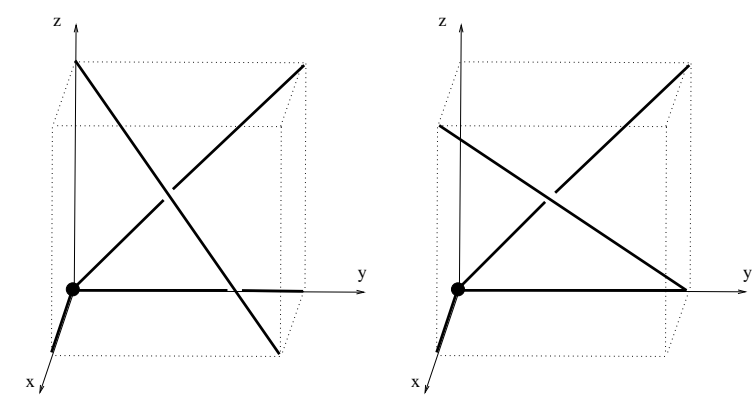

Figure 1. Some motifs of type aafd. 
The resulting 2 model nets, $\mathcal{M}_{1}$ and $\mathcal{M}_{2}$ are equivalent by a rotation about the line through the centre of the cube in the $x$-axis direction. Thus $\mathcal{M}_{1}$ and $\mathcal{M}_{2}$ are equivalent to the model net $\mathcal{M}_{\text {aad }}^{g}$ for the word $a_{x} a_{y} g_{x} d_{1}$.

(iii) Assume that exactly 1 of the 4 edges of $F_{e}$ is an axial edge, which we may assume lies in the $x$-axis. If the 3 remaining edges are the $f$-edges that are incident to the origin, then the transformation of $\mathcal{M}$ by the map $(x, y, z) \rightarrow(x-z, y, z)$ has type aaad and so is equivalent to $\mathcal{M}_{\mathrm{pcu}}^{d}$. If the 3 remaining $f$ edges are not of this form then they are either coplanar (and, as before, $\mathcal{M}$ is equivalent to $\mathcal{M}_{\text {pcu }}^{f}$ ) or only 1 of these 3 edges is incident to the origin, as in Figure 2. In these cases $\mathcal{M}$ is equivalent to a model net with 2 axial edges and so the previous arguments suffice.

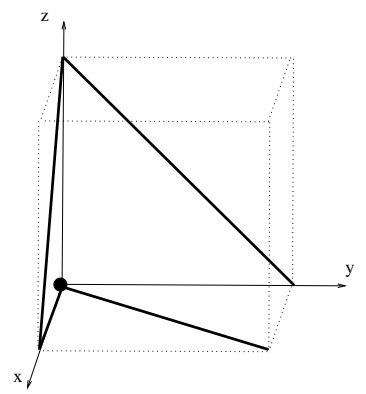

Figure 2. A motif with 3 non coplanar facial edges.

Thus we may assume that the defining word for $\mathcal{M}$ is of type affd,afgd or aggd. Moreover by rotational and translational equivalence we may assume that the possible types are $a_{x} f f d_{1}, a_{x} f g d_{1}$ or $a_{x} g g d_{1}$. If all four edges are incident to the origin then $\mathcal{M}$ is equivalent by an elementary affine transformation to a model net with 2 axial edges and so there are no new cases to consider. Also if 3 edges are incident to the origin then once again the net is equivalent to the net for hex, and so it remains to consider the cases $a_{x} g_{x} g_{y} d_{1}, a_{x} g_{x} g_{z} d_{1}$ and $a_{x} g_{y} g_{z} d_{1}$ indicated in Figure 3 .

Note that the first and third nets are the nets $\mathcal{M}_{\text {ad }}^{g g}$ and $\mathcal{M}_{\text {ad }}^{g_{y} g_{z}}$ in the list of model nets. That these nets are not isomorphic follows from their topological density counts. The second net has a rotation about the diagonal which is a mirror image of the first net and so is equivalent to it by elementary transformations.

(iv) Finally, for the case $m=4$, we assume that there are no axial edges. By rotational symmetry there are 4 cases which, under the convention are uniquely specified by the words $f f f d, f f g d, f g g d$ and $g g g d$. The last of these corresponds to a disconnected net, as we have seen in the previous section, the first gives an alternative model net for ilc (as we have remarked prior to the proof), and the other 2 nets, for $f f g d$ and $f g g d$, are easily seen to be affinely equivalent to a model net with 1 axial edge.

The case $m=5$. It is straightforward to see that if $\mathcal{M}$ has 3 axial edges and 2 face edges then it is equivalent to the model net $\mathcal{M}_{\mathrm{pcu}}^{f f}$ for bct. Also, type aaafd is equivalent 

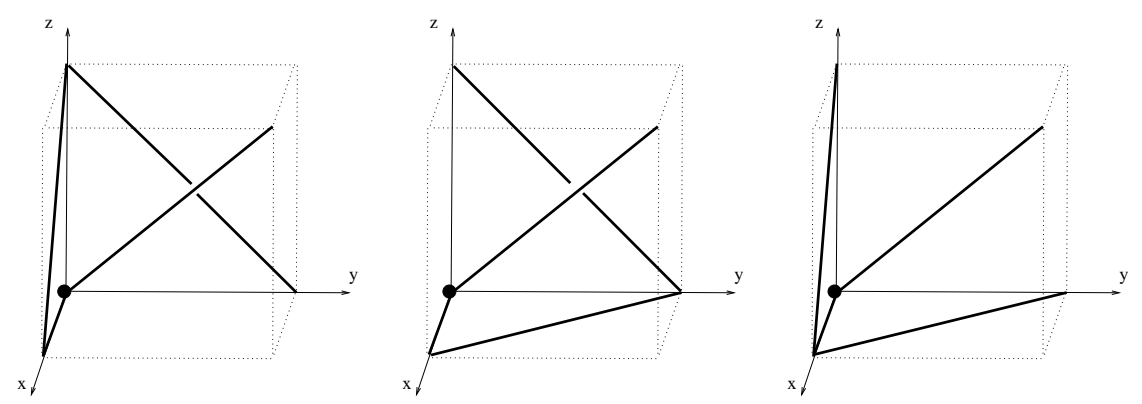

Figure 3. Motifs for the model nets $\mathcal{M}\left(a_{x} g_{x} g_{y} d_{1}\right), \mathcal{M}\left(a_{x} g_{x} g_{z} d_{1}\right)$ and $\mathcal{M}\left(a_{x} g_{y} g_{z} d_{1}\right)$.

to this type. On the other hand, type aaagd has hxl-multiplicity equal to 1, rather than 2 , and so is in a new equivalence class, also with no edge penetrations. In fact this model net has topology ile.

Consider next the model nets with 2 axial edges and no diagonal edges. These also have no penetrating edges and are of hxl-multiplicity 1 or 2 . Moreover it is straightforward to show that each is equivalent by elementary affine transformations to a model net with 3 axial edges and so they equivalent to the model nets for bct and ile respectively. The same is true for the 9 nets of type aawd where $w$ is a word in 2 facial edges which is not of type $g g$.

Thus, in the case of 2 axial edges it remains to consider the types $a_{x} a_{y} w d_{1}$ with $w=$ $g_{x} g_{y}, g_{x} g_{z}$ and $g_{y} g_{z}$ each of which has a penetrating edge of type $4^{2}$. The first two of these are model nets in the list and give new and distinct affine equivalence classes in view of their penetration type and differing $\operatorname{hxl}(\mathcal{N})$ count. The third net, for the word $a_{x} a_{y} g_{y} g_{z} d_{1}$ is a mirror image of the first net and so is orientedly affinely equivalent to it.

It remains to consider the case of 1 axial edge, $a_{x}$, together with $d_{1}$ and 3 facial edges. If there are 2 edges of type $f_{x}, f_{y}$ or $f_{z}$ then there is an elementary equivalence with a model net with 2 axial edges. The same applies if there is a single such edge. For an explicit example consider $a_{x} f_{x} g_{y} g_{z} d_{1}$. The image of this net under the transformation $(x, y, z) \rightarrow(x, y-z, z)$ gives a depth 1 net with 2 axial edges. The transformation of motifs is indicated in Figure 4.
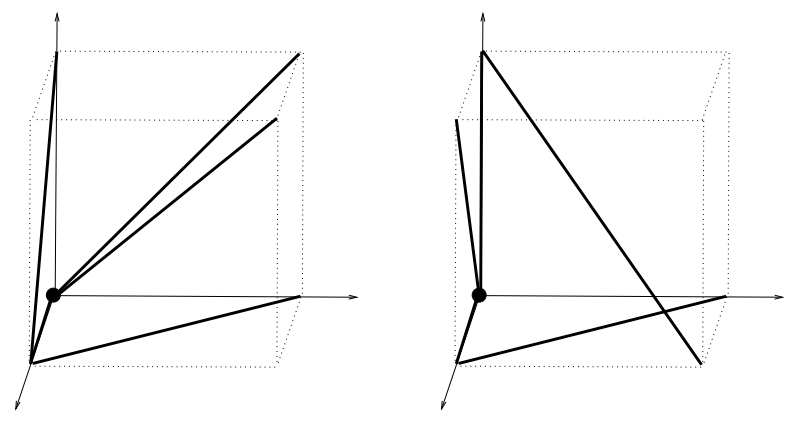

Figure 4. Change of motif under $(x, y, z) \rightarrow(x, y-z, z)$. 
Finally the model net for $a_{x} g_{x} g_{y} g_{z} d_{1}$ appears in the listing and gives a new affine class with penetration type $3^{2}$.

The case $m=6$. We first assume that there is no diagonal edge in the motif for $\mathcal{M}$ and therefore no edge penetration of type $4^{2}$ or $3^{2}$. There are 2 distinguished model nets in the list for this case, one with the 3 facial edges of type $f$ (a net with topology ild) and one where the 3 facial edges are of type $g$ (a net with topology fcu). Two other choices of facial edges are possible (up to rotation) and these are readily seen to be equivalent to the ild and fcu nets.

We may now assume that there exists a diagonal edge in the standardised form of the edge word defining $\mathcal{M}$. If there are 3 axial edges then there are 3 possibilities, namely types aaaffd,aaafgd,aaaggd. The first 2 cases are not new, since the transformation $(x, y, z) \rightarrow(x, y-z, z)$ give motifs without a diagonal edge, while the model net for aaaggd appears in the list, with penetration type $4^{2}$ and $\operatorname{hxl}(\mathcal{M})=2$.

We may now assume that $\mathcal{M}$ has a standardised word $a_{x} a_{y} w d_{1}$ where $w$ is a word in 3 facial edges. For $w$ of $f f f$ type there are 3 cases, namely $f_{x} f_{y} g_{z}, f_{x} g_{y} f_{z}$ and $g_{x} f_{y} f_{z}$, each of which transforms by an elementary transformation (respectively, $x \rightarrow x-z, y \rightarrow y-z$ and $x \rightarrow x-z)$ to a case with 3 axial edges. For $w$ of type $f g g$ there are 3 cases, namely $f_{x} g_{y} g_{z}$, $g_{x} f_{y} g_{z}$ and $g_{x} g_{y} f_{z}$. The first and second of these are not new, since the transformations $y \rightarrow y-z$ and $x \rightarrow x-z$, respectively, lead to an equivalence with $\mathcal{M}_{\mathrm{pcu}}^{g g d}$, while the third case is the model net $\mathcal{M}_{\text {aad }}^{g g f}$.

Finally, for $w$ of type $g g g$ we have the model net $\mathcal{M}_{\text {aad }}^{g g g}$.

The case $m=7$. There are 4 cases of standardised edge word of the form aaawd with $w$ of type $f f f, f f g, f g g$ or $g g g$. The model net for $a_{x} a_{y} a_{z} f_{x} f_{y} f_{z} d$ is obtained from the model net for $a_{x} a_{y} a_{z} f_{x} f_{y} g_{z} d$ by the transformation $y \rightarrow y-z$ followed by a rotation. Thus there is a maximum of 3 equivalence classes with representative model nets $\mathcal{M}_{\mathrm{pcu}}^{f f f d}, \mathcal{M}_{\mathrm{pcu}}^{\text {ggf }}, \mathcal{M}_{\mathrm{pcu}}^{\text {gggd }}$. Since these are distinguished by their edge penetration type the proof is complete. 